\title{
Facial Feature Model for Emotion Recognition Using Fuzzy Reasoning
}

\author{
Renan Contreras, Oleg Starostenko, Vicente Alarcon-Aquino, \\ and Leticia Flores-Pulido \\ CENTIA, Department of Computing, Electronics and Mechatronics \\ Universidad de las Américas Puebla, Cholula, 72820, México \\ \{renan.contrerasgz, oleg.starostenko, \\ vicente.alarcon, leticia.florespo\} @udlap.mx
}

\begin{abstract}
In this paper we present a fuzzy reasoning system that can measure and recognize the intensity of basic or non-prototypical facial expressions. The system inputs are the encoded facial deformations described either in terms of Ekman's Action Units (AUs) or Facial Animation Parameters (FAPs) of MPEG-4 standard. The proposed fuzzy system uses a knowledge base implemented on knowledge acquisition and ontology editor Protégé. It allows the modeling of facial features obtained from geometric parameters coded by AUs - FAPs and also the definition of rules required for classification of measured expressions. This paper also presents the designed framework for fuzzyfication of input variables for fuzzy classifier based on statistical analysis of emotions expressed in video records of standard Cohn-Kanade's and Pantic's MMI face databases. The proposed system has been tested in order to evaluate its capability for detection, classifying, and interpretation of facial expressions.
\end{abstract}

Keywords: Emotion recognition, facial features, knowledge-based framework, rules-based fuzzy classifier.

\section{Introduction}

The fuzzy systems and their combination with neural networks have been successfully used for pattern recognition and for image indexing and interpretation $[1,2]$. In the area of facial expression recognition the application of a fuzzy reasoning remains marginal despite that some researchers have successfully used classifying systems which emulate the way as humans identify prototypical expression [3, 4, 5]. Usually the facial expression recognizing systems are based on two parts: a module for generation of feature vector corresponding to emotion expression in analyzed image (described by pixel position, colors, shapes, regions, etc.) and a classification module that recognize an emotion and describe its intensity.

There are a lot of techniques that have been used for facial features extraction. Some of them are based on Gabor Wavelets [5], Active Appearance and Geometric Models [6], Principal Components Analysis and Hierarchical Radial Basis Function Network [7], Optical Flow and Deformable Templates [8], Discrete Cosine Transform and Neural Networks [9], Multilevel Hidden Markov Models [10], Dynamic Bayesian 
networks [11], and others. Even though these approaches may extract and interpret the facial actions, there are not reports about how they may link standard facial actions with particular formal models or rules for automatic facial expression recognition.

In this paper we present the development of a fuzzy reasoning system, which is able not only to recognize facial expressions using standard Ekman's AUs (Action Units), FAPs (Facial Animation Parameter) and FDPs (Facial Definition Parameter) of MPEG-4 standard, but also to measure their intensity [12]. This proposal permits to create novel models for precise facial feature detection as well as recognition and interpretation of basic and non-prototypical emotions. The proposed fuzzy system for facial expression recognition consists of two principal modules. The first one is a knowledge-based framework for modeling facial deformations by FAP and AU action units [13] developed according to well-known standards [14, 15]. The second module is used for recognizing facial expressions by fuzzy classifier in combination with Gaussian functions providing measurement of emotion intensity. To reduce relative subjectivity and lack of psychological meaning of emotional intensity levels the statistical analysis of facial actions in Cohn-Kanade's and Pantic's image databases has been implemented [16, 17]. This information has been incorporated into the knowledge-based framework enabling to model each facial deformation. The proposed approach has not been widely used in emotion classifiers, but we believe that this technique permits to develop knowledge-base frameworks for facial expression recognition because the analysis of semantics of facial actions may be implemented by using the rule-based descriptors and fuzzy reasoning classifier.

\section{Knowledge-Based Framework}

The knowledge-based framework allows measuring facial deformations in terms of distances between fiducial points modeled by FAPs and AUs and represented by rulebased descriptors used then in the process of fuzzyfication and interpretation of emotion intensity. The fiducial points represented by FDPs of MPEG-4 standard provides the automatic normalization of measured facial deformations making it invariant to scale of input images. The framework also allows modeling facial deformations defining a set of rules for indexing and quantification of expressions.

The proposed approach is able to detect and measure any type of facial expression however it has been tested using six basic expressions (happiness, sadness, disgust, surprise, anger, and fear). Fig. 1 shows the structure of knowledge-based framework that supports design of a fuzzy reasoning system. We exploit relationships between the measured facial deformations and their mathematical description, by the corresponding AUs and FAPs and rules required for identification of expressions. This knowledge-based framework has been implemented using ontology editor Protégé that provides extensible, flexible, and plug-and-play environment that allows fast prototyping and application development [18]. The proposed knowledge-based framework consists of two abstract super-classes: the Face_Model and the Emotion_Model. The Face_Model class defines different approaches for representation of face features. 


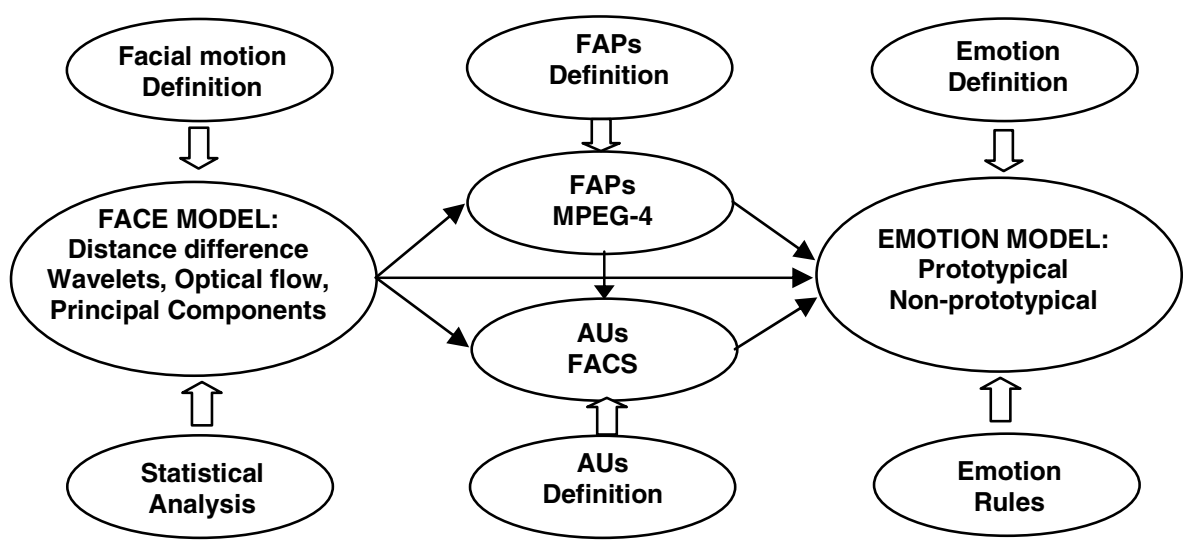

Fig. 1. Structure of emotion knowledge database based on FAPs and AUs

For the framework four specific classes based on AUs, FAPs, FDPs, and FDPs have been created. The Emotion_Model class permits to create the rule-based models for emotion indexing using classes of the Face_Model. The instances of the particular Face_Model class contain the basic facial actions (AUs, FAPs) that include action number, its name, description, direction of motion, involved facial muscles, part of a face where action is occurred, etc. Particularly, we propose emotion indexing based on measuring standard spatial variations of FDP positions implemented by our Distance_Model. The framework may be extended with new non-standard classes and models. The advantage of the proposed framework is that the classes and instances with attributes represent knowledge about emotions, and parameters of any model may be automatically converted to parameters of each other.

\section{The Proposed Facial Model}

In the proposed fuzzy reasoning system the facial model based on analysis of nineteen FDPs has been adopted. It describes all necessary facial actions defining either basic or non-prototypical emotions [13]. Fig. 2 (left) shows the selected FDPs with corresponding number of associated FAPs. Some FDPs are reference points which are remained static during facial deformation. Used FDPs define the Distance_Class that represent distances between fiducial reference points and particular FDPs (see Fig. 2). The facial action parameter FAP represents facial changes of emotional expression with respect to the neutral expression. The difference called $D d$ quantifies facial changes in terms of units defined by MPEG-4 standard. Table 1 shows the fifteen instances of the DistanceClass chosen for our model, geometric definitions of these distances, the measurement units, the relations with FAPs, and the actions, which they describe. Some reports of Plutchik [12], Pantic, [5], and Esau [4] suggest a geometrical model of face which includes not only distances but also angles between the lines connecting the standard FDPs. However this approach does not contribute significant precision and makes the processing too complex $[13,17]$. 

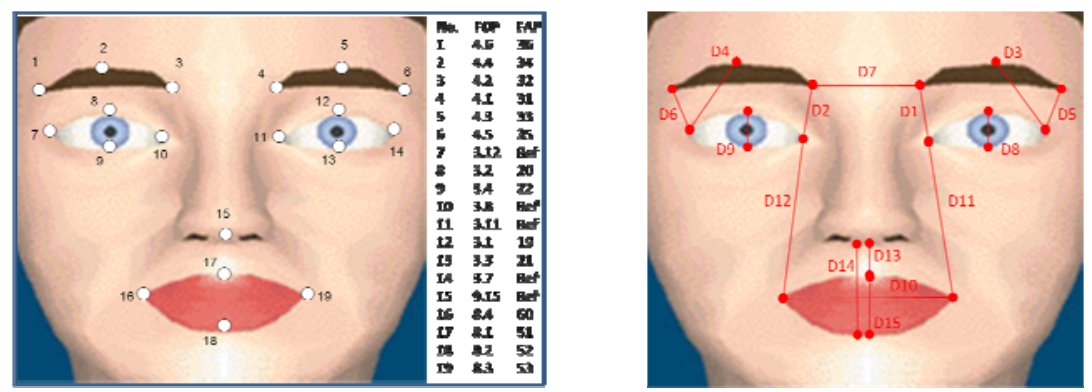

Fig. 2. FDPs used for recognizing facial expressions and definition of Distance_Class instances

Table 1. Description of instances in the DistanceClass

\begin{tabular}{ccccc}
\hline Dd & FDPs Difference & Units & FDP & Action Description \\
\hline D1 & $\mathrm{d}\{3.11,4.1\}$ & ENS & 31 & raise $1 \mathrm{i}$ eyebrow \\
D2 & $\mathrm{d}\{3.8,4.2\}$ & ENS & 32 & raise $\mathrm{i}$ eyebrow \\
D3 & $\mathrm{d}\{3.7,4.3\}$ & ENS & 33 & raise $1 \mathrm{~m}$ eyebrow \\
D4 & $\mathrm{d}\{3.12,4.4\}$ & ENS & 34 & raise $\mathrm{r} \mathrm{m}$ eyebrow \\
D5 & $\mathrm{d}\{3.7,4.5\}$ & ENS & 35 & raise 1 o eyebrow \\
D6 & $\mathrm{d}\{3.12,4.6\}$ & ENS & 36 & raise $\mathrm{r}$ o eyebrow \\
D7 & $\mathrm{d}\{4.1,4.2\}$ & ES & & squeeze $1 / \mathrm{r}$ eyebrow \\
D8 & $\mathrm{d}\{3.3,3.1\}$ & IRISD & $21-19$ & close $\mathrm{t} / \mathrm{b}$ l eyelid \\
D9 & $\mathrm{d}\{3.4,3.2\}$ & IRISD & $22-20$ & close $\mathrm{t} / \mathrm{b}$ r eyelid \\
D10 & $\mathrm{d}\{8.3,8.4\}$ & MW & $53-54$ & stretch $1 / \mathrm{r}$ cornerlip \\
D11 & $\mathrm{d}\{3.11,8.3\}$ & ENS & 59 & raise 1 cornerlip o \\
D12 & $\mathrm{d}\{3.8,8.4\}$ & ENS & 60 & raise $\mathrm{r}$ cornerlip o \\
D13 & $\mathrm{d}\{9.15,8.1\}$ & MNS & & lower $\mathrm{t}$ midlip \\
D14 & $\mathrm{d}\{9.15,8.2\}$ & MNS & & raise $b$ midlip \\
D15 & $\mathrm{d}\{8.1,8.2\}$ & MNS & $51-52$ & raise $b / t$ midlip \\
\hline
\end{tabular}

\section{Fuzzyfication of Distance Instances}

A fundamental process of fuzzy reasoning is fuzzyficacion of input variables and definition of the corresponding membership functions used for indexing facial deformations. The input variables are FAPs representing variation of distances between fiducial points that compose standard database of indexed facial expressions, particularly from Kanade's and Pantic's databases [16, 17]. Each database consists of approximately 500 records with expression of different emotions by 100 subjects in frontal position. Accompanying meta-data include annotation of FAC action units and emotion specified expressions. Recorded videos show a series of 23 facial muscle motions that are described by combination of action units (e.g. AU1+AU2 means inner and outer brows raised). Each record begins from a neutral or nearly neutral emotion (neutral face) finishing on a required target emotion. Table 2 shows the results of quantification of the distance differences (see Fig. 2) between fiducial points describing maximum and minimum values, mean, and standard deviation for 
Table 2. AUs parameters determined for Kanade's database

\begin{tabular}{|c|c|c|c|c|c|}
\hline FACs & Distance & Maximum & Minimun & Mean & Deviation \\
\hline \multirow{2}{*}{ AU1 } & D1 & 339.02 & & 120.55 & 84.52 \\
\hline & D2 & 383.92 & & 123.44 & 88.42 \\
\hline \multirow{2}{*}{ AU2 } & D5 & 190.23 & & 72.16 & 60.02 \\
\hline & D6 & 172.25 & & 35.08 & 67.66 \\
\hline \multirow{7}{*}{ AU4 } & D1 & & -264.20 & -42.54 & 90.92 \\
\hline & D2 & & -243.63 & -38.47 & 92.88 \\
\hline & D3 & & -176.41 & -31.23 & 68.42 \\
\hline & D4 & & -125.68 & -6.20 & 61.99 \\
\hline & D5 & & -120.53 & -35.26 & 43.40 \\
\hline & D6 & & -137.58 & -29.92 & 53.46 \\
\hline & D7 & & -216.24 & -67.69 & 65.20 \\
\hline \multirow{2}{*}{ AU5 } & D8 & 429.14 & & 129.51 & 221.11 \\
\hline & D9 & 474.65 & & 136.61 & 243.04 \\
\hline \multirow{2}{*}{ AU7 } & D8 & & -677.76 & -288.97 & 171.72 \\
\hline & D9 & & -511.21 & -318.66 & 148.63 \\
\hline AU10 & D13 & & -294.11 & -171.46 & 85.75 \\
\hline \multirow{3}{*}{ AU12 } & D10 & 517.28 & & 273.19 & 147.06 \\
\hline & D11 & & -267.11 & -129.71 & 103.15 \\
\hline & D12 & & -268.58 & -140.29 & 122.95 \\
\hline \multirow{2}{*}{ AU15 } & D11 & 438.04 & & 116.17 & 125.59 \\
\hline & D12 & 526.43 & & 118.10 & 152.28 \\
\hline AU16 & D14 & 668.44 & & 306.39 & 247.81 \\
\hline \multirow{2}{*}{ AU20 } & D10 & 345.04 & & 208.07 & 116.20 \\
\hline & D15 & 528.24 & & 282.48 & 144.23 \\
\hline AU25 & D15 & 2248.76 & & 676.64 & 577.28 \\
\hline \multirow{2}{*}{ AU27 } & D10 & & -230.91 & -108.40 & 62.52 \\
\hline & D15 & 2248.76 & & 1440.71 & 401.93 \\
\hline
\end{tabular}

each one associated with the particular AU. Recall that the difference in distances is measured between a neutral face and one with any action expressing an emotion. The similar results have been obtained after analysis of emotion representation by AUs using either Kanade's or Pantic's database.

From Table 2 we already have quantitative definition of action units, which may be used for continuous interpretation of emotion. For measuring intensity of facial expression the Gaussian function has been used applying the following equation (1):

$$
f(x, \sigma, c)=e^{\frac{-(x-c)^{2}}{2 \sigma^{2}}}
$$

The used parameters are determined by mentioned statistical analysis, where $c$ defines the position of peak and $\sigma$ controls the width of the bell shaped Gaussian curve.

The fuzzyfication process may be explained analyzing a behavior of any action unit, for example, AU12. According to the results of statistical analysis made for AU12 (see Table 2) the range of its distance variable, for example, D10 is between 0 and 517.20 MWs (Mouth Width). We have defined for each variable as for all ones 
a)
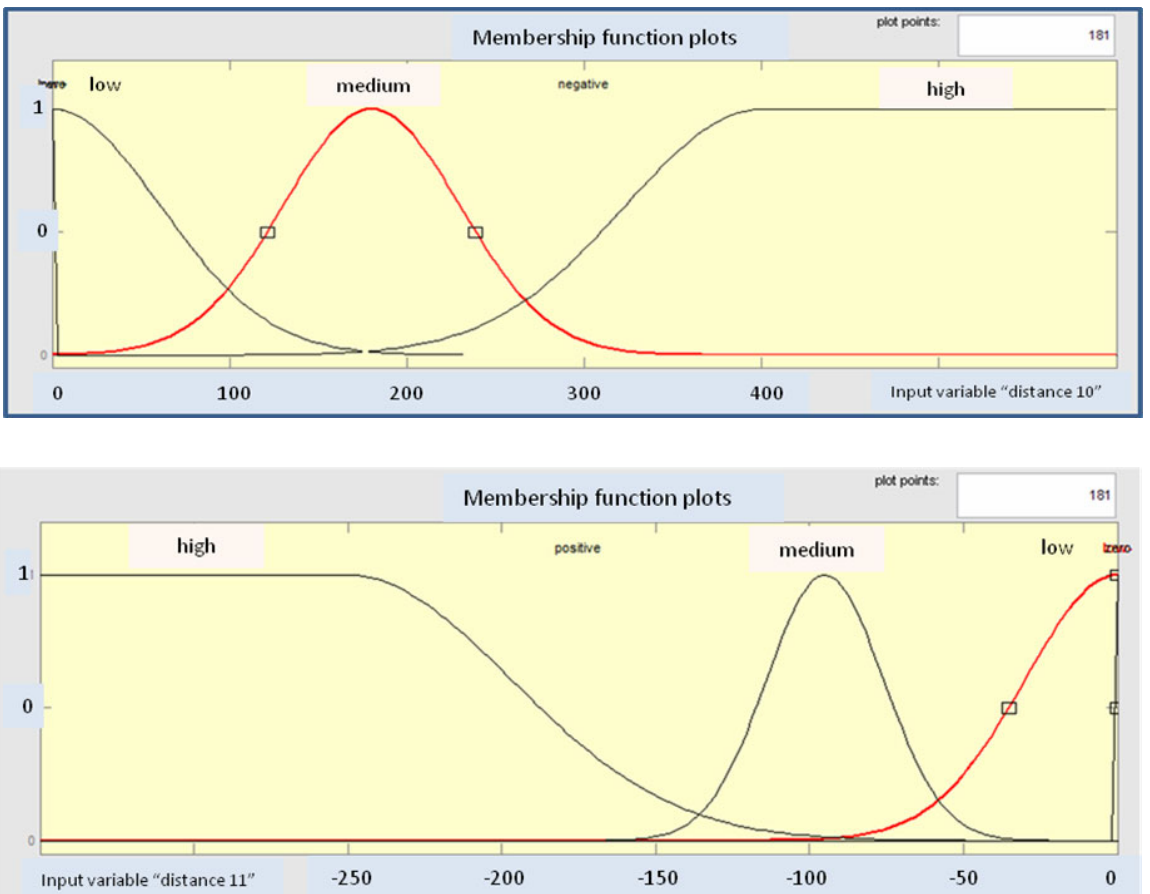

b)

Fig. 3. Membership function plots and intensity partitions for variable a) D10 and b) D11

three levels of emotion intensity (low + , medium+, and high+) dividing in the corresponding proportion the range of distance variation. These intervals may be computed using the data from Table 2 such as center and width of medium section, the mean, and deviation of D10. Having already defined the middle section, then we compute the Gaussian functions for low and high sections. Additionally, a saturation level is included taking into account the maximum possible value of a facial deformation. Fig. 3 a) and b) show the final process of fuzzyfication for variables D10 and D11. The membership functions are obtained for each partition using Gaussian functions providing measurement of intensity of action unit in continuous manner.

\section{Fuzzy Inference System}

The proposed model for fuzzyfication of facial features has been tested on designed fuzzy inference system. The designed system (see Fig.4) consists of two modules: the first module measures value of AUs composing analyzed emotion; the second one recognizes and interprets the intensity of facial expressions. A set of rules defined for fuzzy logic that recognizes and measures intensity of AUs and corresponding emotion is shown in Tables 3 and 4. 


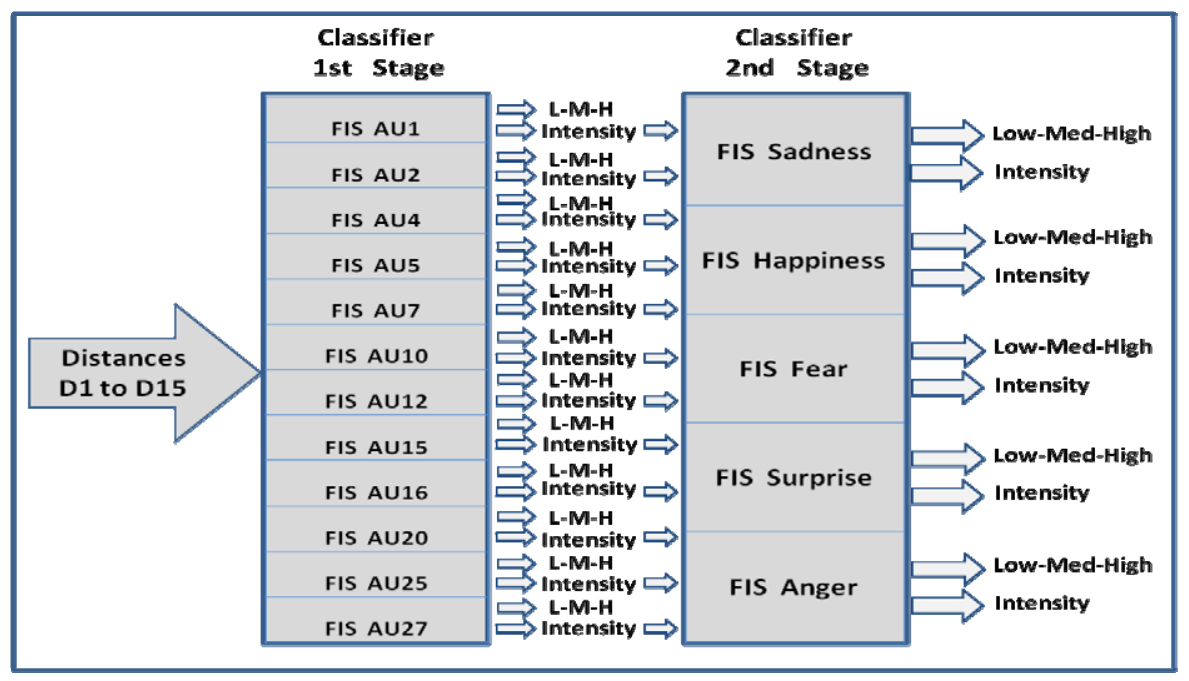

Fig. 4. Block diagram of fuzzy classifier of prototypical emotions

Table 3. Rules and Distance variables for recognizing AUs

\begin{tabular}{lccc}
\hline Code & Description & Distance Diff. & Recognition Rules \\
\hline AU1 & Inner Brow Raiser & D1, D2 & Both increase in same proportion \\
AU2 & Outer Brow Raiser & D5, D6 & Both increase in same proportion \\
AU4 & Brow Lowerer & D3, D4, D7 & D3\&D4 increase, D7 decrease \\
AU5 & Upper Lid Raiser & D8, D9 & Both increase in same proportion \\
AU7 & Lid Tightener & D8, D9 & Both decrease in same proportion \\
AU10 & Upper Lip Raiser & D13 & D13 decrease \\
AU12 & Lip Corner Puller & D10,D11,D12 & D10 increase D11 \& D12 decrease \\
AU15 & Lip Corner Depressor & D11, D12 & Both increase in same proportion \\
AU16 & Lower Lip Depressor & D14 & D14 increase \\
AU20 & Lip stretcher & D10, D11, D12 & D10, D11\&D12 increase \\
AU25 & Lips part & D15 & D15 increase \\
AU27 & Mouth Stretch & D10, D15 & D10 decrease, D15 increase \\
\hline
\end{tabular}

Table 4. Rules and AUs for recognizing facial expressions

\begin{tabular}{|c|c|c|}
\hline Emotion & AUs Used & Recognition Rules \\
\hline Sadness & AU1, AU4, AU15 & Increasing 3 actions increase expression intensity \\
\hline Happiness & AU12, AU7 & $\begin{array}{l}\text { Presence of AU12 \& AU7 but not AU7 (blinking). } \\
\text { Increasing values increase expression intensity }\end{array}$ \\
\hline Fear & $\begin{array}{l}\text { AU1, AU2, AU4, } \\
\text { AU5, AU20,AU27 }\end{array}$ & $\begin{array}{l}\text { Presence of the } 6 \text { actions but not AU7 (blinking). } \\
\text { Increasing values increase expression intensity }\end{array}$ \\
\hline Surprise & $\begin{array}{l}\text { AU1, AU2 AU5 } \\
\text { AU27 }\end{array}$ & $\begin{array}{l}\text { Presence of the 4th action but not AU5 (blinking). } \\
\text { Increasing values increase expression intensity }\end{array}$ \\
\hline Anger & AU4, AU7 & $\begin{array}{l}\text { Presence of AU4 \& AU7 but not AU7 (blinking). } \\
\text { Increasing values increase expression intensity }\end{array}$ \\
\hline
\end{tabular}


In Fig. 5 the user interface of designed fuzzy inference system is shown. In the right upper corner the reasoning process is visualized with intensity of analyzed action unit AU12. The intensity of the input values is tested by classifier applying three discrimination levels described by Gaussian functions: 1-st row in Fig. 5 presents low intensity for all input distances, 2-nd row presents medium and 3-rd high intensity. The shaded areas correspond to magnitude of the membership functions that describe the contribution of each distance difference to particular emotion.

In some cases the displacement of symmetrical points on a face is different. Thus, it is also measured and shown in 4-th column. The intensity of output variables for the particular action unit presented in 5-th column is modeled by three grades described by the triangular functions instead of Gaussian. This approach is easy to implement and provides fast facial expression recognition without additional errors during interpretation. The proposed model of reasoning is flexible enough to allow its extension incorporating new features for recognition of non-prototypical emotions.

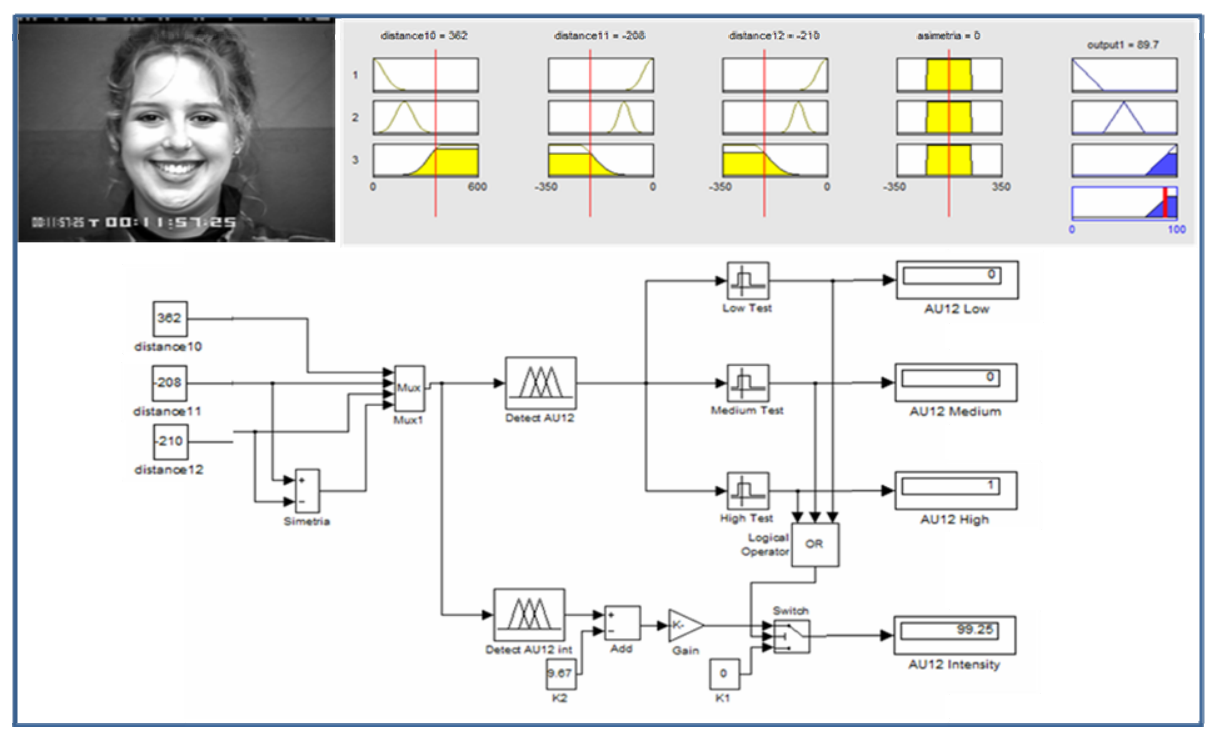

Fig. 5. Measurement of AU12 Lip Corner Puller representing happiness of high intensity

\section{Obtained Results and Discussion}

The test of system performance and efficiency of fuzzyfication model has been done using Kanade's and Pantic's databases. Tables 5 and 6 show the confusion matrices obtained for five basic prototypical emotions in case of medium and high intensity. Finally, with regard to correct evaluation of the expression reported by the system, Table 7 shows the comparison between the intensity of expression Surprise given by the classifier and reported by evaluation committee of ten persons participated in 
Table 5. Confusion Matrix with expression of medium intensity

\begin{tabular}{cccccc}
\hline Emotion & Sadness & Surprise & Happiness & Anger & Fear \\
\hline Sadness & $81 \%$ & $9.50 \%$ & 0 & 0 & $9.50 \%$ \\
Surprise & $0.30 \%$ & $96 \%$ & 0 & 0 & $3.70 \%$ \\
Happiness & 0 & $0.20 \%$ & $96 \%$ & $1.90 \%$ & $1.90 \%$ \\
Anger & 0 & $4.50 \%$ & $0.10 \%$ & $92 \%$ & $3.40 \%$ \\
Fear & $6 \%$ & $5.80 \%$ & 0 & 0 & $88.20 \%$ \\
\hline
\end{tabular}

Table 6. Confusion Matrix for expressions of high intensity

\begin{tabular}{cccccc}
\hline Emotion & Sadness & Surprise & Happiness & Anger & Fear \\
\hline Sadness & $84 \%$ & $8 \%$ & 0 & 0 & $8 \%$ \\
Surprise & $0.20 \%$ & $96.40 \%$ & 0 & 0 & $3.40 \%$ \\
Happiness & 0 & 0 & $97.60 \%$ & $1.20 \%$ & $1.20 \%$ \\
Anger & 0 & $1.70 \%$ & 0 & $96.70 \%$ & $1.60 \%$ \\
Fear & $4.70 \%$ & $5.70 \%$ & 0 & 0 & $89.60 \%$ \\
\hline
\end{tabular}

Table 7. Usability test results for Surprise emotion

\begin{tabular}{|c|c|c|c|c|c|c|c|}
\hline & Output & Evaluation & Status & & Output & Evaluat. & Status \\
\hline 1 & 6.814 & Low & OK & 11 & 51.03 & Medium & $\mathrm{OK}$ \\
\hline 2 & 50.33 & Medium & $\mathrm{OK}$ & 12 & 47.7 & Medium- & $\mathrm{OK}$ \\
\hline 3 & 51.04 & Low & FAIL & 13 & 6.678 & Low & OK \\
\hline 4 & 48.59 & Medium & $\mathrm{OK}$ & 14 & 50.2 & Medium & $\mathrm{OK}$ \\
\hline 5 & 49.85 & Medium & $\mathrm{OK}$ & 15 & 17.95 & Medium & FAIL \\
\hline 6 & 94.08 & High & $\mathrm{OK}$ & 16 & 95.12 & High & $\mathrm{OK}$ \\
\hline 7 & 69.97 & High & OK & 17 & 94.05 & High & OK \\
\hline 8 & 51.46 & Medium & OK & 18 & 49.29 & Medium & OK \\
\hline 9 & 93.93 & High & $\mathrm{OK}$ & 19 & 93.21 & High & $\mathrm{OK}$ \\
\hline 10 & 94.94 & High & $\mathrm{OK}$ & 20 & 93.41 & High & $\mathrm{OK}$ \\
\hline \multicolumn{4}{|c|}{ Correct assessment : } & \multicolumn{4}{|c|}{$90 \%$} \\
\hline
\end{tabular}

usability tests. The obtained results indicate a correct assessment of the intensity about $90 \%$ for Surprise emotion. For other expressions such as joy, sadness, anger, and fear the percentage of corresponding correct recognition is about 80,85, 77, and $75 \%$ respectively. Comparing the results of facial expression recognition with other well-known systems the proposed approach gives average value about $79 \%$ against $72 \%$ of Esau [4]. The high degree of recognition mainly depends on the number of AUs or FAPs used for description of emotion. The recognition of non-prototypical emotions lies in the range about of $40-60 \%$. This low level of recognition is because of complexity in selection of AUs for representation of non-prototypical emotion and due to subjectivity of its perception by each person. The proposed framework opens new possibility for design of systems for emotion detection and intensity recognition. 


\section{Conclusions}

In this paper we presented a model for fuzzyfication of facial features used for recognition of basic or non-prototypical emotions. For quantification of emotions and their intensities a statistical analysis of Kanade's and Pantic's face database has been made. Two-stage fuzzy inference using Gaussian and triangular functions is applied providing measurement of facial expression intensity. In the preliminary experiments the basic emotion recognition achieves up to $70-90 \%$ that depends on complexity in selection of AUs for representation of particular emotion and subjectivity of its perception by each person. The designed knowledge-base framework is general enough to create the diverse instances of emotions, as well as it provides quite exact quantitative description of measured facial actions. This permits simple and formal definition of relationship between emotions, facial actions, and their descriptors. The proposed framework also allows the postulation of rules for prototypical or nonprototypical facial expression recognition using any type of classifiers.

Acknowledgments. This research is sponsored by Mexican National Council of Science and Technology, CONACyT, Projects: \#109115 and \#109417.

\section{References}

1. Young-Joong, K., Myo-Taeg, L.: Near-Optimal Fuzzy Systems Using Polar Clustering: Application. In: Khosla, R., Howlett, R.J., Jain, L.C. (eds.) KES 2005. LNCS (LNAI), vol. 3684, pp. 518-524. Springer, Heidelberg (2005)

2. Yamakawa, T.: Stabilization of an inverted pendulum by a high-speed fuzzy logic controller hardware system, J. Fuzzy Sets and Sys. 32(2), 161-180 (1989)

3. Mufti, M., Khanam, A.: Fuzzy Rule Based Facial Expression Recognition, Computational Intelligence for Modeling, Control and Automation (2006)

4. Esau, N., Wetzel, E. L.: Real-Time Facial Expression Recognition Using a Fuzzy Emotion Model. In: IEEE Fuzzy Systems Conf., pp. 1-6 (2007)

5. Pantic, M.: An Expert System for Multiple Emotional Classification of Facial Expressions. In: 11th IEEE Int. Conf. on Tools with Artif. Intel., p. 113 (1999)

6. Akamatsu, L.S.: Coding facial expressions with Gabor wavelets. McGraw Hill, N.Y. (1998)

7. Kyoung, S.C., Yong-Guk, K., Yang-Bok, L.: Real-Time Expression Recog. Using Active Appearance Model. In: Int. Conf. Comp. Intelligence and Security, China, pp. 1-8 (2006)

8. Lin, D.T.: Facial Expression Classification Using PCA and Hierarchical Radial Basis Function Network. J. Inf. Science and Eng. 22, 1033-1046 (2006)

9. Black, M.J.: Recognizing Facial Expressions in Image Sequences Using Local Parameterized Models of Image Motion. J. of Comp. Vision 25(1), 23-48 (1998)

10. Kharat, G.U., Dudul, S.V.: Neural Network Classifier for Human Emotion Recognition. In: 1-st Int. Conf. on Emerging Trends in Eng. and Techn., Iran, pp. 1-6 (2008)

11. Cohen, I., Garg, A., Huang, T.S.: Emotion Recognition from Facial Expressions using Multilevel HMM. Neural Information Processing Systems, London (2000)

12. Plutchik, R.: The nature of emotions. J. American Scientist 89, 344 (2001)

13. Contreras, R., Starostenko, O.: A Knowledge-base Framework for Analysis of Facial Expressions. In: 10th Conf. on Pat. Recog. and Inf. Proces., Belarus, pp. 251-256 (2009) 
14. Ekman, P., Friesen, W.: Facial Action Coding System (FACS). Consulting Psychologists Press, Palo Alto (1978)

15. ISO/IEC14496-2:2001(E), International Standard, Information technology - Coding of audio-visual objects - Part 2, 2nd Ed. (2001)

16. Kanade T., Cohn J.: Comprehensive database for facial expression analysis. In: 4-th IEEE Conf. on Autom. Face and Gesture Recog. France, pp. 46-53 (2000)

17. Pantic, M., Valstar, M.F., Rademaker, R.: Web-based Database for Facial Expression Analysis. In: IEEE Conf. Multmedia and Expo., Netherlands, pp. 1-6 (2005)

18. Ontology editor Protégé (2009), http: / / protege. stanford. edu 